OPEN ACCESS

Edited by:

Jane Mellor,

University of Oxford, United Kingdom

Reviewed by:

Tomas J. Ekström, Karolinska Institute (KI), Sweden

Thomas Roeder,

University of Kiel, Germany

*Correspondence:

Oscar Yanes

oscar.yanes@urv.cat

Specialty section:

This article was submitted to Epigenomics and Epigenetics,

a section of the journal

Frontiers in Genetics

Received: 18 March 2019

Accepted: 18 June 2019

Published: 05 July 2019

Citation:

Miro-Blanch J and Yanes O (2019)

Epigenetic Regulation at the Interplay Between Gut Microbiota and Host

Metabolism. Front. Genet. 10:638.

doi: 10.3389/fgene.2019.00638

\section{Epigenetic Regulation at the Interplay Between Gut Microbiota and Host Metabolism}

\author{
Joan Miro-Blanch ${ }^{1,2}$ and Oscar Yanes ${ }^{1,2 *}$ \\ ${ }^{1}$ Metabolomics Platform, IISPV, Department of Electronic Engineering, Universitat Rovira i Virgili, Tarragona, Spain, \\ 2 Spanish Biomedical Research Center in Diabetes and Associated Metabolic Disorders (CIBERDEM), Madrid, Spain
}

Gut microbiota communities have coevolved for millions of years in a symbiotic relationship with their mammalian hosts. Elucidating and understanding the molecular mechanisms by which microbiota interacts with its host and how this contributes to the homeostasis of the host is crucial. One of these molecular relationships is the so-called chemical crosstalk between microbiota and host metabolisms, including the poorly explored epigenetic regulation of host tissues by the metabolic activity of gut microbiota in response to changes in diet. DNA methylation and histone modifications are epigenetic marks partly regulated by enzymes such as methylases and acetylases, whose activity depend on host and microbiota metabolites that act as substrates and cofactors for these reactions. However, providing a complete mechanistic description of the regulatory interactions between both metabolisms and the impact on the expression of host genes through an epigenetic modulation, remains elusive. This article presents our perspective on how metabolomic, metagenomic, transcriptomic, and epigenomic data can be used to investigate the "microbiota-nutrient metabolism-epigenetics axis." We also discuss the implications and opportunities this knowledge may have for basic and applied science, such as the impact on the way we structure future research, understand, and prevent diseases like type 2 diabetes or obesity.

Keywords: microbiota, epigenetics, metabolomics, metabolism, histones, omics

\section{CROSSTALK BETWEEN HOST AND GUT MICROBIOTA METABOLISMS}

The human body co-habit with a diverse community of symbiotic microorganisms and their set of genes, collectively known as the microbiome (Ursell et al., 2013). The acquisition of the initial microbiome is a dynamic rather than a static process during early life (Ferretti et al., 2018). A recent estimation of the number of bacterial cells over human cells in our body has reduced the ratio from 10:1 (Savage, 1977) to a 1.3:1 (Sender et al., 2016a; Sender et al., 2016b). This implies a similar number of bacterial and human cells in and on the human body. However, the human microbiome encodes for at least 100 times more genes than our genome (Qin et al., 2010). Therefore, the corresponding higher functionality of bacterial genes is a key aspect to understand existing metabolic interactions between the host and its microbiota.

The microbiota helps their hosts to digest dietary fiber; produces some important neurotransmitters (Sampson and Mazmanian, 2015; Yano et al., 2015; Strandwitz et al., 2018), hormones, and vitamins 
(Kau et al., 2011; Trial et al., 2016); helps in training the host immune system (Kau et al., 2011; Chu and Mazmanian, 2013); and protects against pathogens (Watanabe et al., 2017), among many other functions. However, unbalanced microbiota can also cause disease. Some common diseases in western societies such as obesity and type 2 diabetes are associated with shifts in the relative abundance of gut bacteria composition and functionality, compared to the ones observed in healthy individuals. The cause of the microbiota imbalance (dysbiosis) of unhealthy individuals across age and geography has been mainly correlated with dietary habits (Turnbaugh et al., 2008; De Filippo et al., 2010; Muegge et al., 2011; Wu et al., 2011; Arumugam et al., 2011; Menni et al., 2017). Therefore, the so-called chemical crosstalk between the microbiota and its host has tangible consequences for the physiological state of the host (Sharon et al., 2014; Caesar et al., 2015; Ussar et al., 2016). However, the molecular mechanisms by which microbiota chemically interacts with host cells and regulate gene expression remain largely unknown. In this regard, the role that certain hostmicrobiota derivate metabolites may exert on epigenetic events at the DNA, RNA, and histone level needs to be further investigated.

\section{THE "MICROBIOTA-NUTRIENT METABOLISM-HOST EPIGENETIC" AXIS}

Differences in the microbiota or epigenome in two genetically identical organisms, such as same-sex inbred mice or monozygous twins, can create differences in susceptibilities to diseases including obesity and type 2 diabetes (Ling and Groop, 2009; Franks and Ling, 2010). As with gut microbiota, new studies have demonstrated that epigenetic events are highly dynamic, changing in response to nutrient availability (Bouchard et al., 2010; Hullar and Fu, 2014) or physical exercise (Laker et al., 2017). DNA methyltransferases, DNA hydroxylases, histone acetyltransferases, histone deacetylases, histone methyltransferases, and histone demethylases are enzymes responsible for adding or removing these dynamic epigenetic modifications. In this regard, endogenous metabolites can regulate gene expression through epigenetic events in host cells (Katada et al., 2012). For instance, histone deacetylation regulated by sirtuin family deacetylases is regulated by the $\mathrm{NAD}^{+} / \mathrm{NADH}$ ratio, acetyl-CoA, O-acetylADP-ribose, and nicotinamide (Peleg et al., 2016; Ringel et al., 2018). Whether gut microbiota metabolism is regulating the concentration and/or activity of endogenously produced metabolites by the host remains largely unexplored, and it is only recently that an increasing number of researchers have started to investigate this possibility (Krautkramer et al., 2016; Aleksandrova et al., 2017; Romano et al., 2017). Krautkramer and colleagues (2016) have demonstrated that microbial colonization regulates global histone acetylation and methylation in multiple host tissues in a diet-dependent manner.

Short-chain fatty acids (SCFAs) are exclusively produced by the microbial fermentation of dietary carbohydrates, and their abundances are regulated by the composition of the microbiota (Chen et al., 2007; Cai et al., 2011). Importantly, SCFAs, particularly butyrate and acetate, produced by the microbiota, inhibit histone deacetylases (Figure 1) (Maslowski and MacKay, 2011). Increased levels of histone acetylation promote decondensation and relaxation of chromatin, supporting a more transcriptionally active state of chromatin (Bolduc et al., 2017).

Alteration of chromatin state is a possible mechanism by which gut microbiota induces host immune maturation (Cani et al., 2012; Seeley et al., 2018). Recognition of a "self" antigen should not only be limited to mammalian host antigens, but also symbiotic microbiota antigens forming part of the whole human ecosystem in a healthy state. The human leukocyte antigen (HLA) or major histocompatibility complex (MHC) gene system encodes many antigen-presenting proteins, which are essential to recognize and distinguish "self" from "non-self" antigens. Colonization of germ-free mice has demonstrated the capacity of microbiota-specific species to activate MHC class II genes (Umesaki et al., 1995). However, little is known about the immunomodulatory effect of microbial metabolites. A poorly explored possibility is that epigenetically relevant metabolites such as SCFA, highly influenced by microbiota composition, would regulate MHC gene expression by coordinating activity of enzymes that acetylate and methylate histones and DNA allowing chromatin accessibility (Ting and Trowsdale, 2002).

Regulation of DNA and histone methylation may be driven by complex microbiota-host metabolism interactions involving S-adenosyl methionine (SAM), derived from the essential amino acid methionine through diet (Poirier et al., 2001). Folate plays an essential role by re-methylating homocysteine to methionine (Figure 1), thereby ensuring the provision of SAM (Kim, 2005; Krautkramer et al., 2017). In this regard, enzymes that are depleted in obese microbiomes are frequently involved in cofactor and vitamin metabolism (Greenblum et al., 2012), including the production of cobalamin (vitamin B12), pyridoxal phosphate (the active form of vitamin B6), tetrahydrofolate, and folate (Arumugam et al., 2011; Kau et al., 2011; Yatsunenko et al., 2012). Taken together, dysbiosis of microbiota can influence SAM levels and, as a result, alter the methylation status of DNA and histones. Whether dysbiosis of microbiota can alter $\alpha$-ketoglutarate and succinate levels in specific peripheral host tissues, and regulate the rate of DNA demethylation, is a plausible but little explored possibility. Ten-eleven translocation (TET) enzymes are a key family of DNA and histone demethylases that use $\alpha$-ketoglutarate as co-substrate. However, due to the structural similarity with $\alpha$-ketoglutarate, Tets are susceptible to competitive inhibition by fumarate and succinate, causing an increase in histone and DNA methylation levels (An et al., 2017).

Another modification that could be regulated by microbial metabolism is histone phosphorylation. In response to a low ATP/AMP ratio indicative of energy status, the AMP-activated protein kinase (AMPK) can translocate to chromatin and phosphorylate histone H2B (Bungard et al., 2010). Changes in AMPK activity have been reported in obesity, type 2 diabetes, metabolic syndrome, and cardiovascular disease (Kola et al., 2008). Interestingly, germ-free mice were resistant to obesity and insulin resistance that develop after consuming a Westernstyle, high-fat, and sugar-rich diet (Bäckhed et al., 2007). The persistently lean phenotype of germ-free animals was associated with increased skeletal muscle and liver levels of phosphorylated AMPK. It is also tempting to speculate that phosphotransferase systems (PTS) overrepresented in the Western diet microbiomes 


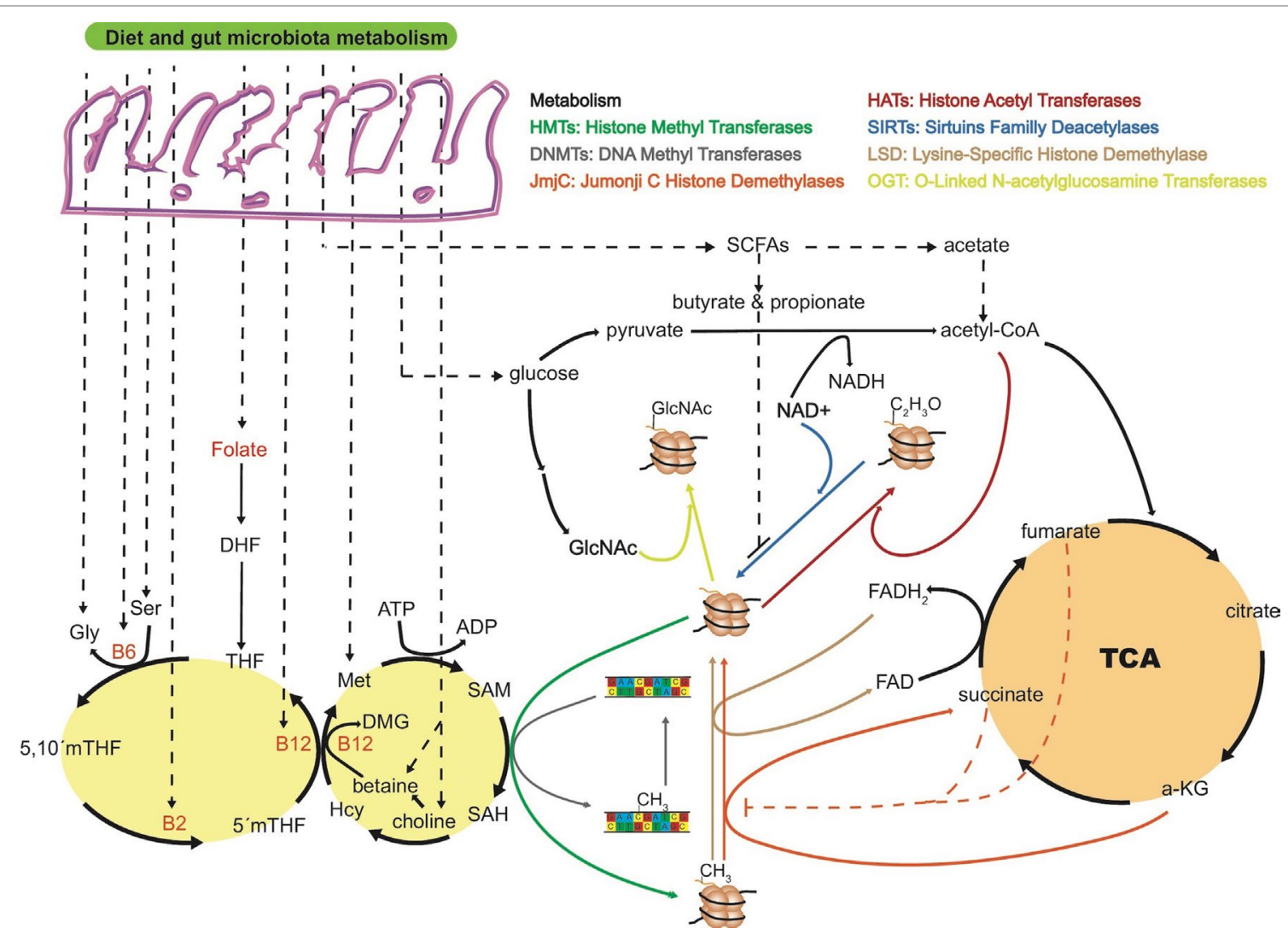

FIGURE 1 | The "microbiota-nutrient metabolism-epigenetics" axis. Most of the key molecules involved in one-carbon metabolism are dietary- and microbiotadependent, being susceptible to gut dysbiosis or diet intervention. Folate is the precursor of dihydrofolate (DHF) and tetrahydrofolate (THF), and dietary intake is the only source for humans. Together with vitamin B12, 5'methy-THF is in charge of remethylating homocysteine (Hcy) to methionine (Met), a crucial step in the process of transferring a methyl group to DNA or histones through SAM. The ratio of S-adenosyl homocysteine (SAH) to SAM regulates the overall methylation status of the genome at the DNA or histone level. Vitamins B12, B2, and B6 are key cofactors in the folate cycle that are produced by the microbiota or ingested through diet. Intermediates of the tricarboxylic acid cycle (TCA) are known to positively or negatively regulate histone methylation. For example, alpha-ketoglutarate $(\alpha-K G)$ is known to be an essential substrate for jumonji $C$ histone demethylases $(\mathrm{mmjC})$, and levels of succinate and fumarate can inhibit jmjC demethylases. $\alpha$-Ketoglutarate is a co-substrate of TET dioxygenases in charge of demethylation processes of histones and DNA. As for jmjC demethylases, increased levels of fumarate and succinate can inhibit TET enzymes with the consequent increased levels of histone and DNA methylation. Short-chain fatty acids (SCFAs) produced by the gut microbiota are also known to inhibit or promote histone PTMs. Butyrate and propionate are inhibitors of sirtuins deacetylases enzymes. Acetate from gut fermentation contributes to the pool of intermediate molecules known to form acetyl-coenzyme A, the major acetyl group donor for histone acetyl transferases (HATS). Acetate is also known to be an inhibitor of histone deacetylases (HDAC), increasing histone acetylation levels and regulating chromatin accessibility. Whether levels of $\mathrm{FAD} / \mathrm{FADH} \mathrm{F}_{2}, \mathrm{NAD} / \mathrm{NADH}, \mathrm{TCA}$ intermediates, and other host endogenous epigenetically relevant metabolites are modulated by gut microbiota metabolism needs to be further investigated. DMG, dimethylglycine; ATP, adenosine triphosphate; ADP, adenosine diphosphate; FAD/FADH ${ }_{2}$, Flavin adenine dinucleotide; $\mathrm{NAD}^{+}$/ $\mathrm{NADH}$, nicotinamide adenine dinucleotide.

(Turnbaugh et al., 2008; Turnbaugh et al., 2009) could have an impact on this histone modification.

In short, dysbiosis and reduction of the microbiota diversity can potentially alter the levels of nutrients and metabolites that can potentially act as regulators of DNA methylation and histone modifications either by directly inhibiting enzymes that catalyze the processes, or by altering the availability of substrates necessary for the enzymatic reactions.

\section{HOLOBIONTS, MULTIFACTORIAL DISEASES, AND OMIC TECHNOLOGIES}

Hosts and their microbiota have a very intimate relationship and should be considered as a single biological and evolutionary unit, termed holobiont (Youle et al., 2013; Carrier and Reitzel, 2017; Groussin et al., 2017; van de Guchte et al., 2018). In this regard, we could arguably talk about holo-genome, -transcriptome, -proteome, or -metabolome, referring to the combination of both, host and host microbiota molecular layers or modules of information at the DNA, RNA, protein, or metabolite level, respectively (Figure 2). To investigate the dynamics of the holobiont ecosystem network, multi-omic approaches bring unprecedented advantages. Diseases such as obesity and diabetes are known to be multifactorial, and the collection of several -omic data (Table 1) from the same holobiont specimen, may provide a detailed molecular description and new mechanistic insights of how dietary nutrients and gut microbiota metabolism can regulate the host phenotype through gene expression and epigenetic and metabolic regulation. 


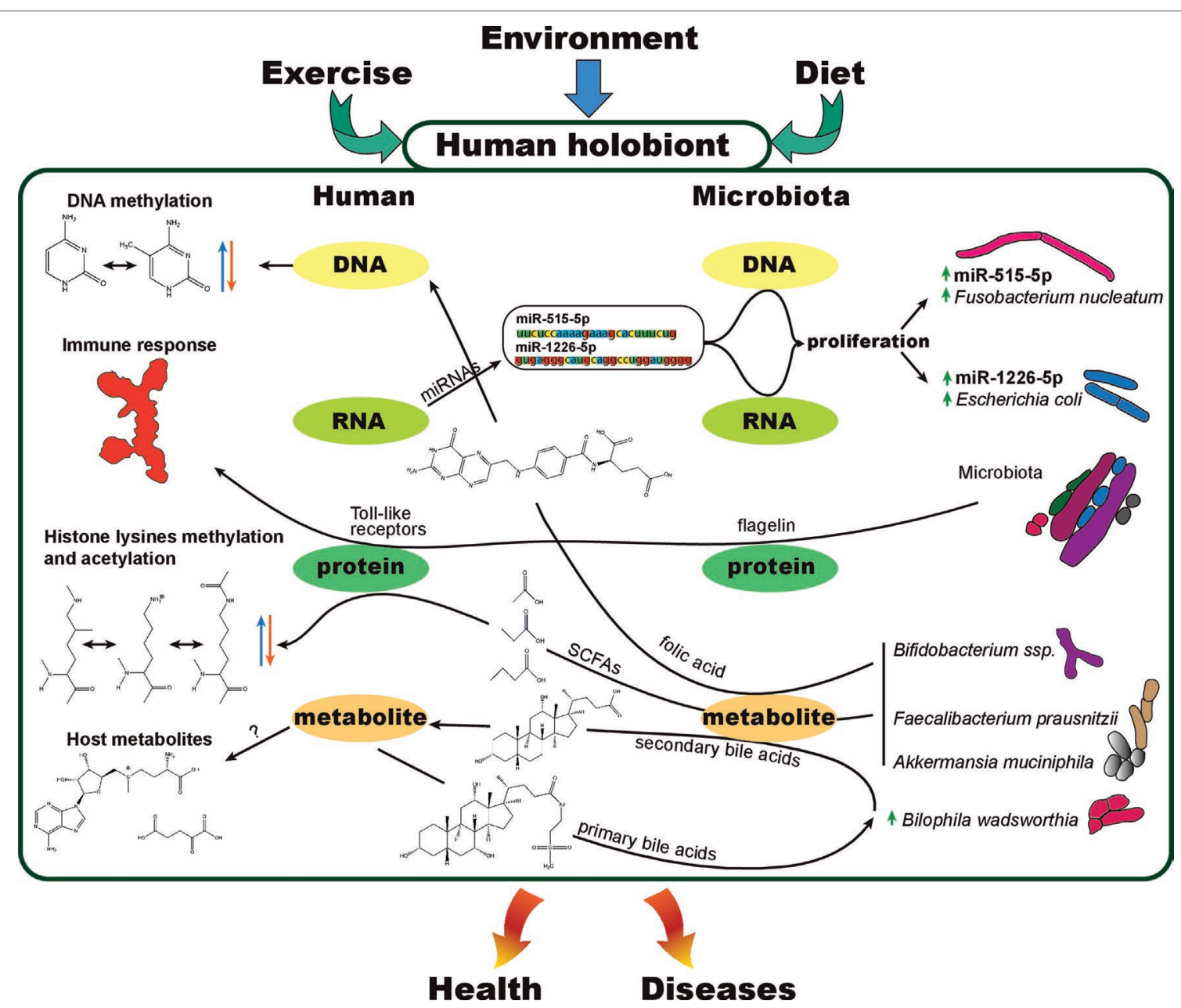

FIGURE 2 | The human holobiont. Representation of few examples of known interactions between different molecular levels within a holobiont. Exercise, environment, and diet can affect the physiology and molecular interactions between human (host) and its microbiota at the DNA, RNA, protein, or metabolite level. As an example, fecal host micro RNAs (miRNAs) are used by the host to modulate the composition of its own gut microbiota, interacting at the microbiota RNA and DNA levels to control microbial growth (Liu et al., 2016). Short-chain fatty acids (SCFAs), products of gut bacterial anaerobic fermentation of dietary fiber, have been proved to cause changes in histone PTMs in multiple host tissues (Krautkramer et al., 2016). Butyrate is a potent histone deacetylase inhibitor (HDACi), regulating the transcription levels of genes involved in colorectal tumorigenesis (Hassig et al., 1997). The direct transformation of dietary nutrients (Sharon et al., 2014) and secondary products of host metabolites such as primary bile acids (Wahlström et al., 2016) evidencies the strong interdependency between host and microbiota. Folate production by Biffidobacterium spp. is another example of how gut microbiota products can affect epigenetics such as DNA or histone methylation (Paul et al., 2015). Microbiota diversity shifts, products, or bacterial structural components such as flagellin can cause the activation of the immune system as well as impact the immune reconstitution after certain diseases or immunotherapy (Manzo and Bhatt, 2015). Immune system maturation and allergic disease development are other examples of how the host and its microbiome interact (Christmann et al., 2015).

To study a complex metabolic disorder such as familial type 1 diabetes mellitus (T1D), Heintz-Buschart et al. (2016) used a combination of host genomics and proteomics together with metagenomics, metatranscriptomics, and metaproteomics to demonstrate a pronounced family membership effect in the structuration and functionality of the microbiomes. They observed a correlation between certain human pancreatic enzymes and the expression of specific microbial genes involved in key T1D metabolic transformations. Krautkramer et al. (2016) used a combination of metabolomic, proteomics of histones, transcriptomic, and metagenomic techniques in conventional and germ-free mice, to demonstrate how SCFAs produced by the microbiota, or supplemented exogenously to germ-free mice, regulate histone post-translational modifications (PTMs). Comparing histone PTMs and transcriptional profiles between conventional, germ-free mice and germ-free mice supplemented with SCFAs, Krautkramer et al. concluded that SCFAs alone are partially causative for histone PTMs. In the same direction, Thaiss et al. (2016) used a combination of metagenomics, transcriptomics, epigenomics, and metabolomics together with imaging electron microscopy, to identify a diurnal rhythmicity in the microbial biogeography, metabolic profile, and metagenomic functionality as critical orchestrators of host epigenetic marks and gene expression. Thaiss et al. have demonstrated that host epigenetic and transcriptional circadian oscillations are partially dependent on environmental signals such as microbiome 
TABLE 1 | Popular omic techniques in the fields of epigenomics, metagenomics, metabolomics, and proteomics.

\begin{tabular}{|c|c|c|c|c|}
\hline Omics & Focus & Trait studied & Techniques used & Reference \\
\hline \multirow[t]{14}{*}{ Epigenomics } & DNA modifications & 5-methylcytosine & WGBS (whole genome bisulfite Seq) & (Lister et al., 2009) \\
\hline & & & RRBS (reduced represented bisulfite Seq) & (Xi et al., 2012) \\
\hline & & & MeDIP-Seq (methylated DNA IP Seq) & (Down et al., 2008) \\
\hline & & 5-hydroxymethylation & oxBS-Seq (oxidative bisulfite Seq) & (Booth et al., 2012) \\
\hline & & 5-formylcytosine & RedBS-Seq (reduced bisulfite Seq) & (Booth et al., 2014) \\
\hline & RNA modifications & 6-methyladenosine & m6A-Seq (m6A specific methylated IP Seq) & (Meyer et al., 2012) \\
\hline & DNA 3D structure & DNA structure and & ChIP-Seq (chromatin IP Seq) & (Barski et al., 2007) \\
\hline & & protein interaction & ATAC-Seq (assay transposase accessible chromatin Seq) & (Buenrostro et al., 2013) \\
\hline & & & Hi-C (chromatin conformation capture) & (Lieberman-Aiden et al., 2009) \\
\hline & & & DNase-Seq (DNase I hypersensitive sites Seq) & (Boyle et al., 2008) \\
\hline & RNA transcripts & Transcribed DNA & RNA-Seq (mRNA/size/strand Seq) & (Mortazavi et al., 2008) \\
\hline & & & GRO-Seq (global run-on-sequencing Seq) & (Core et al., 2008) \\
\hline & & & NET-Seq (native elongating transcript Seq) & (Churchman and Weissman, 2011) \\
\hline & & & UMI method (unique molecular identifiers) & (Kivioja et al., 2012) \\
\hline \multirow[t]{3}{*}{ Metagenomics } & Marker gene & Hypervariable region & 16S gene (16S amplicon PCR/sequencing) & (Woese and Fox, 1977) \\
\hline & Whole metagenome & Whole genome & DNA-Seq (regular DNA Seq) & (Tyson et al., 2004) \\
\hline & Metatranscriptome & RNA & RNA-Seq (regular RNA Seq) & (Gilbert et al., 2008) \\
\hline \multirow[t]{4}{*}{ Metabolomics } & Targeted & Known metabolites & QqQ (triple quadrupole) & (Lu et al., 2008) \\
\hline & Untargeted profiling & Unknown metabolites & qTOF-MS (quadrupole time of flight) & (Patti et al., 2012) \\
\hline & & & Orbitrap-MS & \\
\hline & & & NMR (nuclear magnetic resonance) & (Brindle et al., 2002) \\
\hline \multirow[t]{4}{*}{ Proteomics } & Histones PTMs & $\mathrm{H} 2 \mathrm{~A}, \mathrm{H} 2 \mathrm{~B}, \mathrm{H} 3$, and & Bottom-up & (Sidoli et al., 2012) \\
\hline & & H4 modifications & Middle-down & \\
\hline & & & Top-down & \\
\hline & & & MALDI-imaging mass spectrometry & (Lahiri et al., 2016) \\
\hline
\end{tabular}

The integration and combination of these techniques have the potential to reveal more mechanistic insights on how gut microbiota influence epigenetics and gene expression, ultimately affecting host health. IP, immune precipitation; Seq, sequencing; m6A, 6'methyl adenosine; MS, mass spectrometry; LC, liquid chromatography; GC, gas chromatography; PTMs, posttranslational modifications; MALDI, Matrix-assisted laser desorption ionization.

metabolite dynamics in the intestines and that peripheral organs "sense and adapt" to this circadian metabolite rhythms in a similar manner.

Overall, multi-omic approaches will facilitate the structuration of future research, improve patient stratification toward a more personalized care, and open new avenues to evaluate the effectiveness of functional probiotics, functional foods, or nutritional interventions aimed at regulating host gene expression in health and disease.

\section{OPPORTUNITIES FOR BIOMEDICAL AND CLINICAL RESEARCH}

The development and improvement of new technologies and bioinformatic tools are advancing biomedical research at a fast pace. Multi-omic experiments are allowing researchers to obtain mechanistic insights on the human holobiont homeostasis, improving decision-making for next experiments to be performed. In a research context, extensive collection of -omic data will allow integration of information into modulable and controlled models of microbial communities (Franzosa et al., 2015). However, it is important to identify confounding factors in longitudinal -omic studies. Standardization of methods and techniques to reduce noise and bias in microbiome research will improve how we translate lab findings into the clinic (Knight et al., 2018).

Using germ-free or gnotobiotic mouse models provide a framework to manipulate the gut microbial composition in a controlled manner. These models can be used to study the chemical crosstalk between host and microbiota by uncovering specific epigenetic changes in host cells induced by colonization of specific bacterial strains. Colonizing gnotobiotic mice with single strains or small bacterial consortia, instead of whole fecal transplants, might bring more accurate information to understand specific molecular mechanisms and molecular pathways involved in the host epigenetic regulations. Microbiota-induced dysbiosis with antibiotics might provide a useful approach to validate findings in gnotobiotic models by partially mimicking the effects of absence of microbiota or a reduction in the microbial diversity. Interestingly, organoids might also provide a challenging but very useful in vitro culturing system to study host-microbiota interactions (Williamson et al., 2018).

In a clinical context, gaining knowledge on the "microbiotanutrient metabolism-host epigenetics axis" using multiple -omic approaches in combination with microbiota modulation therapies, has the potential to prevent and treat more efficiently metabolic diseases:

(1) Fecal microbiota transplantation (FMT): The use of microbiota modulation therapies such as FMT to treat recurrent Clostridium difficile infections has been proved significantly more efficient than a vancomycin treatment (Petrof and Khoruts, 2012; van Nood et al., 2013). Recently, the potential of FMT as microbiome modulation technique for treating metabolic (Kootte et al., 2017) (Gupta et al., 2016), neurological (Kang et al., 2017), and immunological disorders (Pamer, 2014) has been tested, improving these conditions by partially restoring microbiota diversity and 
functionality. Assessing long-term host epigenetic effects of FMT has to be further investigated.

(2) Microbiota as drug target: The unique microbiome composition of each person is probably responsible for different susceptibilities to the same nutrient, pollutant, or drug treatment (Koppel et al., 2017). Microbiota-derived metabolites can enter the bloodstream and interact with drug treatments, impacting the efficacy, toxicity, and clearance of the drug. Diagnosing or treating diseases using microbiota-targeted drugs, probiotics, and use of bacteriophages or engineered bacteria has recently re-emerged (Bhat and Kapila, 2017; Landry and Tabor, 2017; Manrique et al., 2017; Maier et al., 2018).

(3) Nutritional intervention, probiotics, and prebiotics: The acute consumption of a cocktail of probiotics containing a selection of five strains of Lactobacillus and five strains of Enterococcus modulates the microbiome and enhances SCFAs production in human and mice (Nagpal et al., 2018). The consumption of some probiotics has proven to be beneficial and ameliorates stress felt in healthy women (Tillisch Kirsten, 2013), improves insulin sensitivity (Gomes et al., 2014), protects against infections (Reid and Burton, 2002), and helps to restore microbiota after distortion by antibiotics (Langdon et al., 2016), among other benefits. Promoting SCFAs producing bacteria in the host gut by a nutritional intervention that increases fiber consumption (Zhao et al., 2018) may have an epigenetic effect in the host (Krautkramer et al., 2016).

\section{CONCLUSIONS AND PERSPECTIVE}

The study of the "microbiota-nutrient metabolism-host epigenetic" axis has great potential to reveal the molecular mechanisms by which gut microbiota composition affects the expression of genes in their hosts. In a human holobiont context, this axis is relevant to understand, prevent, diagnose, and treat the existing epidemic of metabolic disorders such as type 2 diabetes and obesity. Microbiota is a key player in health outcomes due to

\section{REFERENCES}

Aleksandrova, K., Romero-Mosquera, B., and Hernandez, V. (2017). Diet, gut microbiome and epigenetics: emerging links with inflammatory bowel diseases and prospects for management and prevention. Nutrients 9, 1-13. doi: 10.3390/ nu9090962

An, J., Rao, A., and Ko, M. (2017). TET family dioxygenases and DNA demethylation in stem cells and cancers. Exp. Mol. Med. 49, 1-12. doi: 10.1038/ emm. 2017.5

Arumugam, M., Raes, J., Pelletier, E., Le Paslier, D., Yamada, T., Mende, D. R., et al. (2011). Enterotypes of the human gut microbiome. Nature 473, 174-180. doi: 10.1038 /nature09944

Bäckhed, F., Manchester, J. K., Semenkovich, C. F., and Gordon, J. I. (2007). Mechanisms underlying the resistance to diet-induced obesity in germ-free mice. Proc. Natl. Acad. Sci. 104, 979-984. doi: 10.1073/pnas.0605374104

Barski, A., Wei, G., Chepelev, I., Wang, Z., Cui, K., Zhao, K., et al. (2007). Highresolution profiling of histone methylations in the human genome. Cell 129, 823-837. doi: 10.1016/j.cell.2007.05.009

Bhat, M. I., and Kapila, R. (2017). Dietary metabolites derived from gut microbiota: critical modulators of epigenetic changes in mammals. Nutr. Rev. 75, 374-389. doi: 10.1093/nutrit/nux001 the potential myriad of metabolites that can produce and interact with any cell of our body through systemic circulation. Those metabolites are coming from direct transformations of nutrients available in the gut microbiota or from secondary transformed host products. The link between epigenetic marks and gut microbes appears to be mediated by host-microbial metabolites that act as substrates and cofactors for key epigenetic enzymes in the host. A disruption in the composition of the gut microbiota may lead to unbalanced key metabolites that sequentially may impact epigenetic pathways and alter gene expression. The implementation of multi-omic approaches to study the human holobiont will facilitate the stratification of patients toward a personalized-oriented care, improving disease prevention, diagnostics, drug election, and treatment efficiency.

\section{DATA AVAILABILITY}

All datasets analyzed for this study are cited in the manuscript and/or the supplementary files.

\section{AUTHOR CONTRIBUTIONS}

JM and OY designed, wrote, revised, and approved the submitted version of the manuscript.

\section{FUNDING}

This work was supported by the European Union's Horizon 2020 research and innovation program under the Marie SkłodowskaCurie grant agreement No 675610. OY thanks the following bodies for funding: Ministerio de Economia y Competitividad (MINECO) (BFU2017-87958-P) and the Spanish Biomedical Research Centre in Diabetes and Associated Metabolic Disorders (CIBERDEM), an initiative of Instituto de Investigacion Carlos III (ISCIII).
Bolduc, J.-F., Hany, L., Barat, C., Ouellet, M., and Tremblaya, M. J. (2017). Epigenetic metabolite acetate inhibits class I/II histone deacetylases, promotes histone acetylation, and increases HIV-1 Integration in CD4 T cells. J. Virol. 91, 1-15. doi: 10.1128/JVI.01943-16

Booth, M. J., Branco, M. R., Ficz, G., Oxley, D., Krueger, F., Reik, W., et al. (2012). Quantitative sequencing of 5-methylcytosine and 5-hydroxymethylcytosine at single-base resolution. Science (80-). 336, 934-937. doi: 10.1126/science.1220671

Booth, M. J., Marsico, G., Bachman, M., Beraldi, D., and Balasubramanian, S. (2014). Quantitative sequencing of 5-formylcytosine in DNA at single-base resolution. Nat. Chem. 6, 435-440. doi: 10.1038/nchem.1893

Bouchard, L., Rabasa-Lhoret, R., Faraj, M., Lavoie, M. Ė., Mill, J., Pérusse, L., et al. (2010). Differential epigenomic and transcriptomic responses in subcutaneous adipose tissue between low and high responders to caloric restriction. Am. J. Clin. Nutr. 91, 309-320. doi: 10.3945/ajcn.2009.28085

Boyle, A. P., Davis, S., Shulha, H. P., Meltzer, P., Margulies, E. H., Weng, Z., et al. (2008). High-resolution mapping and characterization of open chromatin across the genome. Cell 132, 311-322. doi: 10.1016/j.cell.2007.12.014

Brindle, J. T., McKilligin, E., Tranter, G., Clarke, S., Holmes, E., Nicholson, J. K., et al. (2002). Rapid and noninvasive diagnosis of the presence and severity of coronary heart disease using $1 \mathrm{H}-\mathrm{NMR}$-based metabonomics. Nat. Med. 8, 1439-1445. doi: 10.1038/nm802 
Buenrostro, J. D., Giresi, P. G., Zaba, L. C., Chang, H. Y., and Greenleaf, W. J. (2013). Transposition of native chromatin for multimodal regulatory analysis and personal epigenomics. Nat. Methods 10, 1213-1218. doi: 10.1038/nmeth.2688

Bungard, D., Benjamin, J., Fuerth, Zeng, P.-Y., Faubert, B., Maas, N. L., et al. (2010). Signaling kinase AMPK activates stress-promoted transcription via histone H2B phosphorylation. Science (80-). 329, 1201-1205. doi: 10.1126/ science.1191241

Caesar, R., Tremaroli, V., Kovatcheva-datchary, P., and Patrice, D. (2015). Crosstalk between gut microbiota and dietary lipids aggravates WAT inflammation through TLR signaling. Cell Metab., 22, 658-668. doi: 10.1016/j.cmet.2015.07.026

Cai, L., Sutter, B. M., Li, B., and Tu, B. P. (2011). Acetyl-CoA induces cell growth and proliferation by promoting the acetylation of histones at growth genes. Mol. Cell 42, 426-437. doi: 10.1016/j.molcel.2011.05.004

Cani, P. D., Osto, M., Geurts, L., and Everard, A. (2012). Involvement of gut microbiota in the development of low-grade inflammation and type 2 diabetes associated with obesity. Gut Microbes 3, 37-41. doi: 10.4161/gmic.19625

Carrier, T. J., and Reitzel, A. M. (2017). The hologenome across environments and the implications of a host-associated microbial repertoire. Front. Microbiol. 8, 1-13. doi: 10.3389/fmicb.2017.00802

Chen, Y., Sprung, R., Tang, Y., Ball, H., Sangras, B., Kim, S. C., et al. (2007). Lysine propionylation and butyrylation are novel post-translational modifications in histones. Mol. Cell. Proteomics 6, 812-819. doi: 10.1074/mcp.M700021-MCP200

Christmann, B. S., Abrahamsson, T. R., Charles, N., Duck, L. W., Mannon, P. J., Berg, G., et al. (2015). Human seroreactivity to gut microbiota antigens. J. Allergy Clin. Immunol. 136, 1378-1386. doi: 10.1016/j.jaci.2015.03.036

Chu, H., and Mazmanian, S. K. (2013). Innate immune recognition of the microbiota promotes host-microbial symbiosis. Nat. Immunol. 14, 668-675. doi: 10.1038/ni.2635

Churchman, L. S., and Weissman, J. S. (2011). Nascent transcript sequencing visualizes transcription at nucleotide resolution. Nature 469, 1-18. doi: 10.1038 /nature 09652

Core, L. J., Waterfall, J. J., and Lis, J. T. (2008). Nascent RNA sequencing reveals widespread pausing and divergent initiation at human promoters. Science (80-). 322, 1845-1848. doi: 10.1126/science.1162228

De Filippo, C., Cavalieri, D., Di Paola, M., Ramazzotti, M., Poullet, J. B., Massart, S., et al. (2010). Impact of diet in shaping gut microbiota revealed by a comparative study in children from Europe and rural Africa. Proc. Natl. Acad. Sci. 107, 14691-14696. doi: 10.1073/pnas.1005963107

Down, T. A., Rakyan, V. K., Turner, D. J., Flicek, P., Li, H., Kulesha, E., et al. (2008). A bayesian deconvolution strategy for immunoprecipitation-based DNA methylome analysis. Nat. Biotechnol. 26, 779-785. doi: 10.1038/nbt1414

Ferretti, P., Pasolli, E., Tett, A., Asnicar, F., Gorfer, V., Fedi, S., et al. (2018). Mother-to-infant microbial transmission from different body sites shapes the developing infant gut microbiome. Cell Host Microbe 24, 133-145.e5. doi: 10.1016/j.chom.2018.06.005

Franks, P. W., and Ling, C. (2010). Epigenetics and obesity: the devil is in the details. BMC Med. 8, 88. doi: 10.1186/1741-7015-8-88

Franzosa, E. A., Hsu, T., Sirota-Madi, A., Shafquat, A., Abu-Ali, G., Morgan, X. C., et al. (2015). Sequencing and beyond: integrating molecular "omics" for microbial community profiling. Nat. Rev. Microbiol. 13, 360-372. doi: 10.1038/ nrmicro3451

Gilbert, J. A., Field, D., Huang, Y., Edwards, R. A., Li, W., Gilna, P., et al. (2008). Detection of large numbers of novel sequences in the metatranscriptomes of complex marine microbial communities. PLoS One 3, 277-286. doi: 10.1002/9781118010549.ch27

Gomes, A. C., Bueno, A. A., Souza, R. G. M., and Mota, J. F. (2014). Gut microbiota, probiotics and diabetes. J. Clin. Gastroenterol. 13, 1-13. doi: 10.1097/MCG.0000000000001058

Greenblum, S., Turnbaugh, P. J., and Borenstein, E. (2012). Metagenomic systems biology of the human gut microbiome reveals topological shifts associated with obesity and in flammatory bowel disease. Proc. Natl. Acad. Sci. 109, 594-599. doi: 10.1073/pnas.1116053109

Groussin, M., Mazel, F., Sanders, J. G., Smillie, C. S., Lavergne, S., Thuiller, W., et al. (2017). Unraveling the processes shaping mammalian gut microbiomes over evolutionary time. Nat. Commun. 8, 1-12. doi: 10.1038/ncomms14319

Gupta, S., Allen-Vercoe, E., and Petrof, E. O. (2016). Fecal microbiota transplantation: In perspective. Therap. Adv. Gastroenterol. 9, 229-239. doi: 10.1177/175 $6283 \times 15607414$
Hassig, C. A., Tong, J. K., and Schreiber, S. L. (1997). Fiber-derived butyrate and the prevention of colon cancer. Chem. Biol. 4, 783-789. doi: 10.1016/ S1074-5521(97)90111-3

Heintz-Buschart, A., May, P., Laczny, C. C., Lebrun, L. A., Bellora, C., Krishna, A., et al. (2016). Integrated multi-omics of the human gut microbiome in a case study of familial type 1 diabetes. Nat. Microbiol. 2, 1-12. doi: 10.1038/ nmicrobiol.2016.180

Hullar, M. A. J., and Fu, B. C. (2014). Diet, the gut microbiome, and epigenetics. Cancer J. (United States) 20, 170-175. doi: 10.1097/PPO.0000000000000053

Kang, D. W., Adams, J. B., Gregory, A. C., Borody, T., Chittick, L., Fasano, A., et al. (2017). Microbiota transfer therapy alters gut ecosystem and improves gastrointestinal and autism symptoms: an open-label study. Microbiome 5, 1-16. doi: 10.1186/s40168-016-0225-7

Katada, S., Imhof, A., and Sassone-Corsi, P. (2012). Connecting threads: epigenetics and metabolism. Cell 148, 24-28. doi: 10.1016/j.cell.2012.01.001

Kau, A. L., Ahern, P. P., Griffin, N. W., Goodman, A. L., and Gordon, J. I. (2011). Human nutrition, the gut microbiome and the immune system. Nature 474, 327-336. doi: 10.1038/nature10213

Kim, Y.-I. (2005). Nutritional epigenetics: impact of folate deficiency on dna methylation and colon cancer susceptibility. J. Nutr. 135, 2694-2697. doi: $10.1093 / \mathrm{jn} / 135.11 .2703$

Kivioja, T., Vähärautio, A., Karlsson, K., Bonke, M., Enge, M., Linnarsson, S., et al. (2012). Counting absolute numbers of molecules using unique molecular identifiers. Nat. Methods 9, 72-74. doi: 10.1038/nmeth.1778

Knight, R., Navas, J., Quinn, R. A., Sanders, J. G., and Zhu, Q. (2018). Best practices for analysing microbiomes. Nat. Rev. Microbiol. 16, 410-422. doi: 10.1038/ s41579-018-0029-9

Kola, B., Grossman, A. B., and Korbonits, M. (2008). The role of AMP-activated protein kinase in obesity. Front. Horm. Res. 36, 198-211. doi: 10.1159/000115366

Kootte, R. S., Levin, E., Salojärvi, J., Smits, L. P., Hartstra, A. V., Udayappan, S. D., et al. (2017). Improvement of insulin sensitivity after lean donor feces in metabolic syndrome is driven by baseline intestinal microbiota composition. Cell Metab. 26, 611-619.e6. doi: 10.1016/j.cmet.2017.09.008

Koppel, N., Rekdal, V. M., and Balskus, E. P. (2017). Chemical transformation of xenobiotics by the human gut microbiota. Science (80-). 356, 1246-1257. doi: $10.1126 /$ science.aag2770

Krautkramer, K. A., Kreznar, J. H., Romano, K. A., Vivas, E. I., Barrett-Wilt, G. A., Rabaglia, M. E., et al. (2016). Diet-microbiota interactions mediate global epigenetic programming in multiple host tissues. Mol. Cell 64, 982-992. doi: 10.1016/j.molcel.2016.10.025

Krautkramer, K. A., Rey, F. E., and Denu, J. M. (2017). Chemical signaling between gut microbiota and host chromatin: What is your gut really saying? J. Biol. Chem. 292, 8582-8593. doi: 10.1074/jbc.R116.761577

Lahiri, S., Sun, N., Solis-Mezarino, V., Fedisch, A., Ninkovic, J., Feuchtinger, A., et al. (2016). In situ detection of histone variants and modifications in mouse brain using imaging mass spectrometry. Proteomics 16, 437-447. doi: 10.1002/ pmic. 201500345

Laker, R. C., Garde, C., Camera, D. M., Smiles, W. J., Zierath, J. R., Hawley, J. A., et al. (2017). Transcriptomic and epigenetic responses to short-term nutrientexercise stress in humans. Sci. Rep. 7, 1-12. doi: 10.1038/s41598-017-15420-7

Landry, B. P., and Tabor, J. J. (2017). Engineering diagnostic and therapeutic gut bacteria. Microbiol. Spectr. 5, 1-22. doi: 10.1128/microbiolspec.BAD-0020-2017

Langdon, A., Crook, N., and Dantas, G. (2016). The effects of antibiotics on the microbiome throughout development and alternative approaches for therapeutic modulation. Genome Med. 8, 1-16. doi: 10.1186/s13073-016-0294-z

Lieberman-Aiden, E., Berkum5, van, N. L., Williams, L., Imakaev2, M., Ragoczy, T., et al. (2009). Comprehensive mapping of long range interactions reveals folding principles of the human genome. Science (80-). 326, 289-293. doi: $10.1126 /$ science. 1181369

Ling, C., and Groop, L. (2009). Epigenetics: a molecular link between environmental factors and type 2 diabetes. Diabetes 58, 2718-2725. doi: 10.2337/db09-1003

Lister, R., Pelizzola, M., Dowen, R. H., Hawkins, R. D., Hon, G., Nery, J. R., et al. (2009). Human DNA methylomes at base resolution show widespreadepigenomic differences. Nature 462, 315-322. doi: 10.1038/ nature 08514

Liu, S., Da Cunha, A. P., Rezende, R. M., Cialic, R., Wei, Z., Bry, L., et al. (2016). The host shapes the gut microbiota via fecal MicroRNA. Cell Host Microbe 19, 32-43. doi: 10.1016/j.chom.2015.12.005 
Lu, W., Bennett, B. D., and Rabinowitz, J. D. (2008). Analytical strategies for LC-MS-based targeted metabolomics. J. Chromatogr. B. Analyt. Technol. Biomed. Life Sci. 871, 236-242. doi: 10.1016/j.jchromb.2008.04.031

Maier, L., Pruteanu, M., Kuhn, M., Zeller, G., Telzerow, A., Anderson, E. E., et al. (2018). Extensive impact of non-antibiotic drugs on human gut bacteria. Nature 555, 623-628. doi: 10.1038/nature25979

Manrique, P., Dills, M., and Young, M. J. (2017). The human gut phage community and its implications for health and disease. Viruses 9, 9-11. doi: 10.3390/ v9060141

Manzo, V. E., and Bhatt, A. S. (2015). The human microbiome in hematopoiesis and hematologic disorders. Blood 126, 311-318. doi: 10.1182/blood-2015-04-574392

Maslowski, K. M., and MacKay, C. R. (2011). Diet, gut microbiota and immune responses. Nat. Immunol. 12, 5-9. doi: 10.1038/ni0111-5

Menni, C., Jackson, M. A., Pallister, T., Steves, C. J., Spector, T. D., and Valdes, A. M. (2017). Gut microbiome diversity and high-fibre intake are related to lower long-term weight gain. Int. J. Obes. 41, 1099-1105. doi: 10.1038/ijo.2017.66

Meyer, K. D., Saletore, Y., Zumbo, P., Elemento, O., Mason, C. E., and Jaffrey, S. R. (2012). Comprehensive Analysis of mRNA methylation reveals enrichment in 3' UTRs and near stop codons. Cell 149, 1635-1646. doi: 10.1016/j.cell.2012. 05.003

Mortazavi, A., Williams, B. A., McCue, K., Schaeffer, L., and Wold, B. (2008). Mapping and quantifying mammalian transcriptomes by RNA-Seq. Nat. Methods 5, 621-628. doi: 10.1038/nmeth.1226

Muegge, B. D., Kuczynski, J., Knights, D., Clemente, J. C., Fontana, L., Henrissat, B., et al. (2011). Diet drives convergence in gut microbiome functions across mammalian phylogeny and within humans. Science (80-). 332, 970-974. doi: $10.1126 /$ science. 1198719

Nagpal, R., Wang, S., Ahmadi, S., Hayes, J., Gagliano, J., Subashchandrabose, S., et al. (2018). Human-origin probiotic cocktail increases short-chain fatty acid production via modulation of mice and human gut microbiome. Sci. Rep. 8 , 1-15. doi: 10.1038/s41598-018-30114-4

Pamer, E. G. (2014). Fecal microbiota transplantation: effectiveness, complexities, and lingering concerns. Mucosal Immunol. 7, 210-214. doi: 10.1038/mi. 2013.117

Patti, G. J., Yanes, O., and Siuzdak, G. (2012). Metabolomics: the apogee of the omics trilogy. Nat. Cell Biol. 13, 263-269. doi: 10.1038/nrm3314

Paul, B., Barnes, S., Demark-Wahnefried, W., Morrow, C., Salvador, C., Skibola, C., et al. (2015). Influences of diet and the gut microbiome on epigenetic modulation in cancer and other diseases. Clin. Epigenetics 7, 1-11. doi: 10.1186/s13148-015-0144-7

Peleg, S., Feller, C., Ladurner, A. G., and Imhof, A. (2016). The metabolic impact on histone acetylation and transcription in ageing. Trends Biochem. Sci. 41, 700-711. doi: 10.1016/j.tibs.2016.05.008

Petrof, E. O., and Khoruts, A. (2012). From stool transplants to next-generation microbiota therapeutics. Gastroenterology 1, 233-245. doi: 10.1016/j.dcn.2011. 01.002

Poirier, L. A., Wise, C. K., Delongchamp, R. R., and Sinha, R. (2001). Blood determinations of S -adenosylmethionine, S -adenosylhomocysteine, and homocysteine: correlations with diet. Cancer Epidemiol. Biomarkers Prev. 10, 649-655.

Qin, J., Li, R., Raes, J., Arumugam, M., Burgdorf, S., Manichanh, C., et al. (2010). A human gut microbial gene catalog established by metagenomic sequencing. Nature 464, 59-65. doi: 10.1038/nature08821

Reid, G., and Burton, J. (2002). Use of Lactobacillus to prevent infection by pathogenic bacteria. Microbes Infect. 4, 319-324. doi: 10.1016/S1286-4579(02) 01544-7

Ringel, A. E., Tucker, S. A., and Haigis, M. C. (2018). Chemical and physiological features of mitochondrial acylation. Mol. Cell 72, 610-624. doi: 10.1016/j. molcel.2018.10.023

Romano, K. A., Martinez-del Campo, A., Kasahara, K., Chittim, C. L., Vivas, E. I., Amador-Noguez, D., et al. (2017). Metabolic, epigenetic, and transgenerational effects of gut bacterial choline consumption. Cell Host Microbe 22, 279-290. doi: 10.1016/j.chom.2017.07.021

Sampson, T., and Mazmanian, S. (2015). Control of brain development, function, and behavior by the microbiome. Cell Host Microbe 17, 565-576. doi: 10.1016/j. chom.2015.04.011

Savage, D. D. C. (1977). Microbial ecology of the gastrointestinal tract. Annu. Rev. Microbiol. 31, 107-133. doi: 10.1146/annurev.mi.31.100177.000543
Seeley, J. J., Baker, R., Mohamed, G., Bruns, T., Hayden, M. S., Deshmukh, S. D., et al. (2018). Induction of innate immune memory via microRNA targeting of chromatin remodeling factors. Nature 559, 114-119. doi: 10.1038/ s41586-018-0253-5

Sender, R., Fuchs, S., and Milo, R. (2016a). Are we really vastly outnumbered? Revisiting the ratio of bacterial to host cells in humans. Cell 164, 337-340. doi: 10.1016/j.cell.2016.01.013

Sender, R., Fuchs, S., and Milo, R. (2016b). Revised estimates for the number of human and bacteria cells in the body. PLoS Biol. 14, 1-14. doi: 10.1371/journal. pbio. 1002533

Sharon, G., Garg, N., Debelius, J., Knight, R., Dorrestein, P. C. and Mazmanian, S. K. (2014). Specialized metabolites from the microbiome in health and disease. Cell Metab. 20, 719-730. doi: 10.1016/j.cmet.2014.10.016

Sidoli, S., Cheng, L., and Jensen, O. N. (2012). Proteomics in chromatin biology and epigenetics: elucidation of post-translational modifications of histone proteins by mass spectrometry. J. Proteomics 75, 3419-3433. doi: 10.1016/j. jprot.2011.12.029

Strandwitz, P., Kim, K. H., Terekhova, D., Liu, J. K., Sharma, A., Levering, J., et al. (2018). GABA-modulating bacteria of the human gut microbiota. Nat. Microbiol. 4, 396-403. doi: 10.1038/s41564-018-0307-3

Thaiss, C. A., Levy, M., Korem, T., Dohnalová, L., Shapiro, H., Jaitin, D. A., et al. (2016). Microbiota diurnal rhythmicity programs host transcriptome oscillations. Cell 167, 1495-1510.e12. doi: 10.1016/j.cell.2016.11.003

Tillisch, K., Labus, J., Kilpatrick, L., Jiang, Z., Stains, J., Ebrat, B., et al. (2013). Consumption of Fermented Milk Product with Probiotics Modulates Brain Activity. Gastroenterology 144, 1394-1401. doi: 10.1053/j.gastro.2013.02.043

Ting, J. P. Y., and Trowsdale, J. (2002). Genetic control of MHC class II expression. Cell 109, 21-33. doi: 10.1016/S0092-8674(02)00696-7

Trial, R. D. P., Reijnders, D., Goossens, G. H., Hermes, G. D. A., Zoetendal, E. G., Dejong, C. H. C., et al. (2016). Effects of gut microbiota manipulation by antibiotics on host metabolism in obese humans. Cell Metab. 24, 63-74. doi: 10.1016/j.cmet.2016.06.016

Turnbaugh, P. J., Bäckhed, F., Fulton, L., and Gordon, J. I. (2008). Diet-induced obesity is linked to marked but reversible alterations in the mouse distal gut microbiome. Cell Host Microbe 3, 213-223. doi: 10.1016/j.chom.2008.02.015

Turnbaugh, P. J., Hamady, M., Yatsunenko, T., Cantarel, B. L., Duncan, A., Ley, R. E., et al. (2009). A core gut microbiome in obese and lean twins. Nature 457, 480-484. doi: 10.1038/nature07540

Tyson, G. W., Chapman, J., Hugenholtz, P., Allen, E. E., Ram, R. J., Richardson, P. M., et al. (2004). Community structure and metabolism through reconstruction of microbial genomes from the environment. Nature 428, 37-43. doi: 10.1038/ nature 02340

Umesaki, Y., Okada, Y., Matsumoto, S., Imaoka, A., and Setoyama, H. (1995). Segmented filamentous bacteria are indigenous intestinal bacteria that activate intraepithelial lymphocytes and induce MHC Class II molecules and fucosyl asialo GM1 glycolipids on the small intestinal epithelial cells in the ex-germfree mouse. Microbiol. Immunol. 39, 555-562. doi: 10.1111/j.1348-0421.1995. tb02242.x

Ursell, L. K., Metcalf, J. L., Parfrey, L. W., and Knight, R. (2013). Definig the human microbiome. Nutr. Rev. 70, S38-S44. doi: 10.1111/j.1753-4887.2012.00493.x

Ussar, S., Fujisaka, S., and Kahn, C. R. (2016). Interactions between host genetics and gut microbiome in diabetes and metabolic syndrome. Mol. Metab. 5, 795803. doi: 10.1016/j.molmet.2016.07.004

van de Guchte, M., Blottière, H. M., and Doré, J. (2018). Humans as holobionts: implications for prevention and therapy. Microbiome 6, 81. doi: 10.1186/ s40168-018-0466-8

van Nood, E., Vrieze, A., Nieuwdorp, M., Fuentes, S., Zoetendal, E. G., de Vos, W. M., et al. (2013). Duodenal infusion of donor feces for recurrent clostridium difficile. N. Engl. J. Med. 368, 407-415. doi: 10.1056/NEJMoa1205037

Wahlström, A., Sayin, S. I., Marschall, H. U., and Bäckhed, F. (2016). Intestinal crosstalk between bile acids and microbiota and its impact on host metabolism. Cell Metab. 24, 41-50. doi: 10.1016/j.cmet.2016.05.005

Watanabe, K., Gilchrist, C. A., Uddin, M. J., Burgess, S. L., Abhyankar, M. M., Moonah, S. N., et al. (2017). Microbiome-mediated neutrophil recruitment via CXCR2 and protection from amebic colitis. PLoS Pathog. 13, 1-20. doi: 10.1371/journal.ppat. 1006513

Williamson, I. A., Arnold, J. W., Samsa, L. A., Gaynor, L., DiSalvo, M., Cocchiaro, J. L., et al. (2018). A high-throughput organoid microinjection platform to 
study gastrointestinal microbiota and luminal physiology. Cmgh 6, 301-319. doi: 10.1016/j.jcmgh.2018.05.004

Woese, C. R., and Fox, G. E. (1977). Phylogenetic structure of the prokaryotic domain: the primary kingdoms. Proc. Natl. Acad. Sci. 74, 5088-5090. doi: 10.1073/pnas.74.11.5088

Wu, G. D., Chen, J., Hoffmann, C., Bittinger, K., Chen, Y., Sue, A., et al. (2011). Linking long-term dietary patterns with gut microbial enterotypes. Science (80-). 334, 105-108. doi: 10.1126/science.1208344

Xi, Y., Bock, C., Müller, F., Sun, D., Meissner, A., and Li, W. (2012). RRBSMAP: a fast, accurate and user-friendly alignment tool for reduced representation bisulfite sequencing. Bioinformatics 28, 430-432. doi: 10.1093/bioinformatics/ btr668

Yano, J. M., Yu, K., Donaldson, G. P., Shastri, G. G., Ann, P., Ma, L., et al. (2015). Indigenous bacteria from the gut microbiota regulate host serotonin biosynthesis. Cell 161, 264-276. doi: 10.1016/j.cell.2015.02.047

Yatsunenko, T., Rey, F. E., Manary, M. J., Trehan, I., Dominguez-Bello, M. G., Contreras, M., et al. (2012). Human gut microbiome viewed across age and geography. Nature 486, 222-227. doi: 10.1038/nature11053
Youle, M., Knowlton, N., Rohwer, F., Gordon, J., and Relman, D. A. (2013). Superorganisms and holobionts. Microbe Mag. 8, 152-153. doi: 10.1128/ microbe.8.152.1

Zhao, L., Zhang, F., Ding, X., Wu, G., Lam, Y. Y., Shi, Y., et al. (2018). Gut bacteria selectively promoted by dietary fibers alleviate type 2 diabetes. Sci. 1156, 11511156. doi: $10.1126 /$ science.aao5774

Conflict of Interest Statement: The authors declare that the research was conducted in the absence of any commercial or financial relationships that could be construed as a potential conflict of interest.

Copyright (c) 2019 Miro-Blanch and Yanes. This is an open-access article distributed under the terms of the Creative Commons Attribution License (CC $B Y$ ). The use, distribution or reproduction in other forums is permitted, provided the original author(s) and the copyright owner(s) are credited and that the original publication in this journal is cited, in accordance with accepted academic practice. No use, distribution or reproduction is permitted which does not comply with these terms. 